\title{
Logos, Pathos, and Entertainment
}

\author{
Ryohei Nakatsu \\ Interactive \& Digital Media Instutite, National University of Singapore \\ 21 Heng Mui Keng Terrace, I-Cube Building Level 2, \\ Singapore 119613 \\ idmdir@nus.edu.sg
}

\begin{abstract}
Various new forms of entertainment using information and media technologies have emerged and been accepted among people all over the world. Casual and serious games, as well as communication using mobile phones, blogs, and Twitter, are such kinds of new entertainment. It is important to discuss the basic characteristics of such entertainment and to understand the direction to which these new forms are leading human societies. This paper provides a comparative study of entertainment between developing countries and developed countries, and between ancient times and the present day. The future relationship between entertainment and society is also described.
\end{abstract}

\section{Introduction}

New network, information, and media technologies are rapidly changing our society, including human relationships, lifestyles, and communication. Entertainment is one area that these new technologies are strongly influencing. One good example is games. Playing computer and video games is a common daily activity for people, especially for younger generations. In particular, online games have become very popular in both the U.S. and Asian countries. Another good example is communication. People communicate with their families and friends through e-mail, mobile phones, texting, Twitter, and other means. Until the 1980s, communication media such as telephones were mainly used for business communication. Today, however, communication extends beyond business conversations and has become a form of everyday entertainment.

There are many discussions on these phenomena, but most of them merely observe what is happening in society and report the fact that more people are spending more time on these new forms of entertainment. Unfortunately, however, there has been little consideration of the basic reasons why these new forms have been accepted by people all over the world. One fundamental questions is whether this a totally new phenomenon within our long history. Another question is which aspects of society are changing through the introduction of these new forms of entertainment, and how much those aspects are changing. Another, more difficult question is the direction to which such entertainment is leading our society. In other words, what will be the future of our society, where people are expected to spend more time on entertainment than they do now. 
In this paper, I try to answer these questions by starting from the question of what entertainment is. It will be clarified that over the long course of human history, people have tried to clearly separate logical and emotional aspects of our behaviors, in other words, the logos and pathos, respectively. Consequently, we have succeeded in confining the great power of emotion to only the private aspects of our lives.

New media such as games and mobile phones, however, have strongly affected this relationship between logos and pathos and have partly destroyed it. Today, people are showing the emotional aspect of their behaviors even in formal situations. A more distinctive point is that this tendency is more obvious in Western countries.

Finally, given these considerations, I anticipate the direction to which our society is headed. I also discuss what we should do to maintain our basic identity as human beings.

\section{The Nature of Entertainment}

\subsection{What Is Entertainment?}

At least one billon people currently face starvation all over the world. For these people it is crucial to obtain food for tomorrow or even today. This problem has been one of the most serious topics at global conferences and meetings such as World Economic Forum [1].

On the other hand, in developed countries new types of entertainment have emerged, such as chat on mobile phones and games on game systems and PCs. People in those countries are tending to spend more and more time enjoying such forms of entertainment. There have been significant concerns and complaints about this trend. The basic logic of such complaints is that compared with other human activities, such as education, business, industrial production, and so on, entertainment is not productive. In other words, the complaints suggest that entertainment is only a waste of time.

An important question, however, is whether this is actually true, when the established entertainment industry is huge and includes the movie, game, sports, and other businesses. Another question is why the demand and markets for such entertainment are so huge. The issue of whether entertainment is a waste of time clearly requires care.

\subsection{Origin of Entertainment}

What is happening now is not a totally new phenomenon. In earlier times, human life was simple. Humans farmed or hunted to survive. When people were not occupied with these tasks, they entertained themselves by various means. In other words, we can say that food is strongly related to our physical sustainability, yet at the same time, we can point out that entertainment is related to our mental and spiritual sustainability.

Then the era of civilization began. People introduced various novel types of activities, such as art, business, learning and teaching, and religion. Because of these activities, entertainment came to be considered as a secondary activity in human life. Although entertainment remained a certain part of our everyday life, it has not been considered an essential part. Figure 1 illustrates these changes in our physical and mental sustainability. 


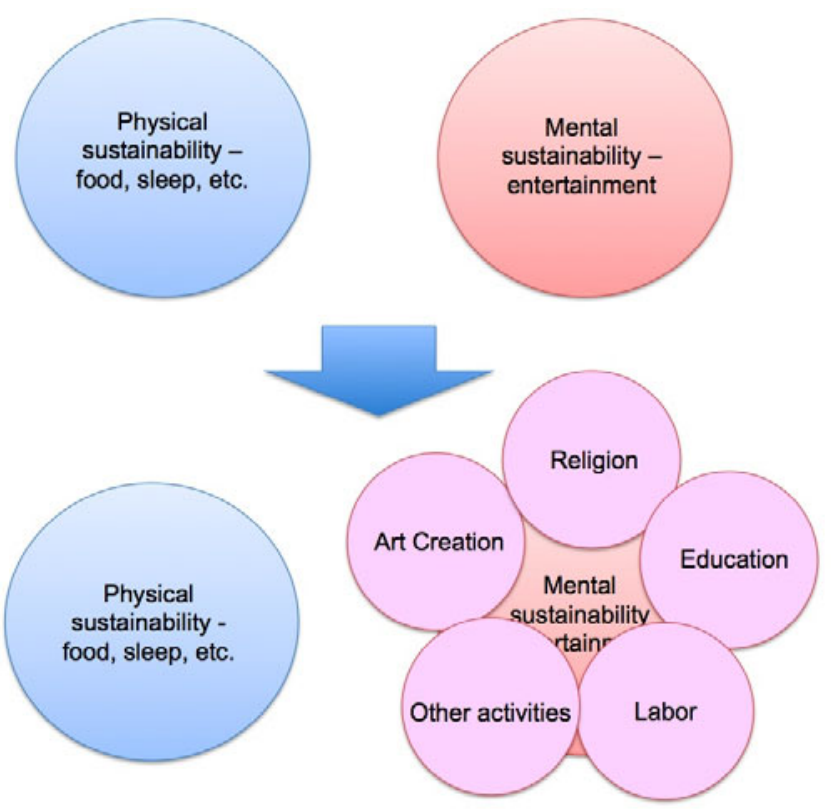

Fig. 1. Changes in the physical and mental sustainability of human beings

\subsection{Our Life and Entertainment}

Even now sometimes that fact that entertainment is an important part of our life becomes apparent. Consider passengers on an airplane. During a flight, most people sleep, eat, or entertain themselves by watching movies, reading novels, and so forth. Only a few people work during a flight. This means that in a simple situation, our lifestyle consists of three basic activities: sleeping, eating, and entertaining ourselves.

What is happening now is, in one sense, an "entertainment renaissance." The introduction of new technologies, especially interactive technologies, into traditional forms of entertainment has totally renewed and strengthened those forms. People are again noticing the basic strength and meaning of entertainment and recognizing that it is a substantial part of their lives. This is a key point in trying to understand such substantial issues as the role of entertainment in developed and developing countries and the future direction of entertainment.

\section{Entertainment in Developing and Developed Countries}

\subsection{Entertainment in Developing Countries}

The importance of entertainment in developing countries has been underestimated. As described above, entertainment has been, from its origin, an essential part of our life. In once sense, its importance is almost second only to survival. Even though few people in developing countries use PCs, mobile phones, or game systems, they intuitively know the importance of entertainment. This is the key point. 
Leaders and academics in the developed world have wondered and struggled with how to introduce a higher level of development into developing countries. For example, it was considered difficult to teach the importance of activities such as education, religion, and business to people facing starvation. What is happening in developed countries, however, can simplify this. In developed countries it is observed that an emerging phenomenon of the merger of entertainment with other activities such as education and business. In other words, many activities commonplace in the developed world are becoming forms of entertainment. This is, in one sense, a fundamental change in business model for various human behaviors and activities. Another important point is that this new business model works even without utilizing cutting-edge technologies.

\subsection{An Entertainment Renaissance}

In other words, the border between entertainment and activities such as education and business is going to disappear. This is why the current phenomenon could be regarded as an "entertainment renaissance."

This means that many serious human activities contain the essence of entertainment and could be enjoyed. This could make it easier to promote such activities as various types of entertainment to people in developing countries. This would lead to greater participation in activities such as education and business, because these activities could now be interpreted as a form of entertainment and would thus be more familiar.

On the other hand, we should carefully examine the new forms of entertainment enthusiastically accepted by younger generations in developed countries. The question is whether these are actually new types of entertainment or not. When we examined new entertainment types in detail, we found that most of them have their origins in older forms of entertainment. For example, the experience of playing role-playing games is almost the same as the experience of reading fantasy novels. Another good example is new types of communication. Communication via chat, texting, or Twitter, using mobile phones, is actually a reshaped form of everyday conversation among family members and friends.

This means that new information and media technologies have reshaped traditional entertainment while keeping the same basic concept. Because of this, while the game market expanded with surprisingly great speed in the early days of video and PC games, it has now apparently reached a certain level of saturation, and various new forms of entertainment face the problem of sustainability. We should probably understand the core concept of entertainment and the future directions of the game industry by studying how people in developing countries entertain themselves.

\section{Logos, Pathos, and Entertainment}

\subsection{Logos and Pathos}

What is happening in developed countries is actually deep and substantial. Through the emergence of new forms of entertainment, our societies and lifestyles are experiencing a fundamental change. 


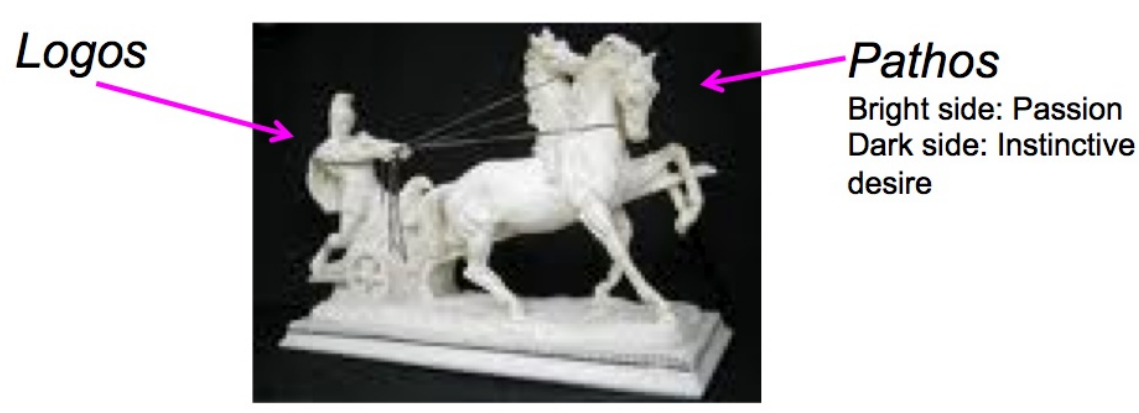

Fig. 2. Logos and pathos

Here, we recall the ancient Greek origin of Western philosophy. Plato compared the human spirit to a carriage with two horses and one driver in his Republic [2]. Here, as illustrated in Fig. 2, the driver is a metaphor for the rational aspect of the human spirit, called the "logos." On the other hand, the two horses are a metaphor for the emotional aspect, called the "pathos." The former could be linked to the formal parts of our lives, and the latter, to the private parts. Furthermore, one of the horses represents passion, while the other represents the instinctive aspect of emotion.

Plato admired logos as the basis of rational human behaviors. As for pathos, passion was admired as the source of creative behaviors, but instinct was despised as undeveloped and representing the dark side of behavior. Plato also expected that logos could control the dark side of pathos, instinct, with help from the bright side of pathos, passion.

This definition and statement by Plato determined the initial direction of philosophy and morality in the Western world. Since the ancient Greek era, people have been trying to separate logos and pathos in their lives. In other words, they have been trying to separate the logical and emotional aspects of living.

\subsection{Formal and Informal Aspects of Life}

In the long history of Western society, starting with Plato's philosophical considerations, logos has been considered related to the formal aspects of human behaviors, in social situations and in business scenes, for example. On the other hand, pathos has been related to the private aspects of human activities. Westerners have long and eagerly sought to clearly separate these two aspects. In addition, people have tried to emphasize the importance of logos while neglecting pathos.

Asian languages also make such a distinction. For example, in Japan the formal aspect of behavior is called honne, while the informal aspect is called tatemae. In terms of honne and tatemae, Japanese people have been accused of having a double standard of behavior. As the above observation of Western society shows, however, this is not correct. All humans have both formal and private aspects of their behavior, in other words, honne and tatemae. The problem is that because people in Asian societies have not been very conscious of these two aspects, they have not been good at clearly separating these two types of behavior. In other words, Japanese people could be said to present confusion of the formal and private aspects of behavior. 
One good example is that of the former prime minster, Mr. Asoh. He once declared at the Japanese parliament that he was actually against privatization of Japan's Post Office when he was a member of the previous Koizumi cabinet. The problem with his behavior is that, because he was a member of the cabinet and this formal decision was made within the cabinet, it was inappropriate to express his private opinion in a formal situation. What was worse was that he thought he was being honest by expressing his private opinion and could not understand why people accused him of expressing honne.

\subsection{Approach between Logos and Pathos}

In contrast with the above situation, the introduction of new media and forms of entertainment has invaded deeply into our lives and changed our behaviors. It was long considered civilized and sophisticated to act logically and hide the emotional aspect of behavior. Now, however, people tend to expose their emotions even in the formal parts of their lives. A good example is means of communication. It has become common for people to communicate even with close friends or family members by using mobile phones or PCs even during meetings or during dinner. This is surprising because communication with people close to us has typically been a private, emotional behavior.

This means that in our everyday life emotional behaviors have again become influential and can play a major role, after long years of separation between logos and pathos and the higher priority of logos over pathos in formal situations. In one sense, our behaviors are returning to those in ancient times. Moreover, this phenomenon has another important aspect. In observing the phenomenon, we notice two fundamental, distinctive features. First, human behaviors in the Western and Eastern worlds are becoming more similar. Second, human behaviors in developed countries and developing countries are also becoming more similar. In other words, differences in human behavior styles between Western and Asian countries, and between developed and developing countries, are disappearing. People are beginning to share the same ways of thinking and cultural principles and rules. In one sense, this is good because it will lower barriers between different people and countries. At the same time, however, we are losing the local features of cultures, which have long been preserved over the course of history.

\section{Media and Entertainment}

\subsection{History of Media}

It is important to consider why we are losing the separation between formal and informal aspects of our behaviors. As indicated above, the arrival of new media strongly influences this phenomenon.

To explain this, consider the history of significant media inventions. The two most impactful media inventions are written characters and the invention of printing typography. These remarkable inventions have enabled people to think, memorize, discuss, and describe by using language. In other words, these inventions have made people more left-brain dependent. It is noteworthy that this mainly happened in Western 
countries, as shown by the long history of Western philosophy, as represented by Plato. What happened in Asian counties is somewhat different. Somehow people did not try to clearly separate rational and emotional actions. The reason for this is an interesting research topic and is discussed elsewhere. In any case, however, for Asian people the concept of separation between logos and pathos has not been so clear.

\subsection{Influence of Movies and Telephones}

Two more impactful inventions in the recent history of media are telephones and movies. Today we tend to focus on recent inventions such as video games, mobile phones, e-mail, blogs, and Twitter. Unfortunately, we have almost forgotten the major impact telephones and movies have had on the basic changes in our behavior.

Before the invention of the telephone, the formal and emotional aspects of our behavior were clearly separated. For emotional behaviors, mental distance and special distance were closely related. When we are together with people to whom we are close, such as family members and friends, we expose our private, emotional behaviors. We have a strong instinct to be connected with familiar people. Before the invention of the telephone, however, when we were spatially separated from such people, because we had no method of communicating with them, we had to hide our emotional aspects and behave formally, as in business situations. Then, after the telephone was invented, it enabled us to be connected to familiar people even though we were spatially separated. Since that time, people have gradually tended to mix the formal and emotional aspects of our lives. This is the fundamental reason why now, even during meetings, dinner, or other situations, people want to continue communicating with people to whom they are close by using mobile phones and smart phones. In other words, the telephone has initiated confusion between the rational and emotional aspects of our behavior. The development of mobile phones and other recent media is only accelerating this trend.

The invention of movies has had a similar effect. Before the invention of movies, people were trained to be left-brain dependent, and reading and writing were the major intellectual and communication behaviors. The invention of movies, however, introduced images as an important communication medium. Images have a strong power to directly influence the emotional part of the brain, the right brain. Therefore, after long years of training to rationally use the left brain, people began to depend more on using the right brain. This means that people have gradually become emotionally dependent instead of rationally dependent. The recent trend toward excessive use of computer graphics and animation originated with the invention of movies. These newer technologies are merely accelerating the trend.

\subsection{Future of Entertainment and Media}

As described above, after long years of separation between logos and pathos in human behavior, these two aspects are moving back together, we can expect that they will merge again in the future, as illustrated in Fig. 3. 


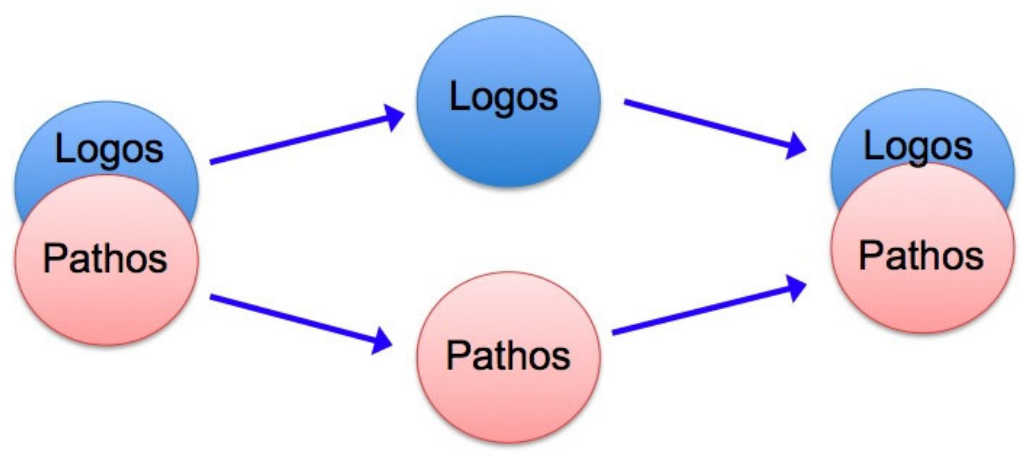

Fig. 3. Transitions of the relationship between logos and pathos

Regarding what this means for the future, it can be pointed out that an interesting and important phenomenon would happen. That is the behaviors of Westerners and Asians could become more alike and even become identical. As described above, in Asian countries a clear separation of logos and pathos has somehow not reached conscious awareness or discussion. Because of this, Asian people have been accused of being underdeveloped as human beings. In Western countries, however, people's behaviors are becoming more like those of Asian people [3]. A good example is communication behaviors. Today, even during such formal events as classes, meetings, discussions, or dinner, people often tend to check e-mail on their mobile phones and try to communicate with people to whom they are close. When a plane lands and arrives at a gate, the first thing many people do is take out their mobile phones, turn them on, and check e-mail or start calling family members. In this regard, there is no distinctive difference between the behaviors of Westerners and Asians.

\subsection{Future of Human Beings}

In one sense, this phenomenon is good, as various gaps between the West and Asia have long been headaches for cultural understanding. We can probably regard this as a bright side of globalization, but on the other side there is a danger. Each country and each ethnicity has developed its own culture, resulting in a rich variety of cultures all over the world. This is a major human accomplishment. The merger of behaviors between the West and Asia, however, could destroy this rich variety of cultural differences among different countries [4]. It is difficult to anticipate a future in which there are no cultural differences and people all over the world are connected to the network all the time and repeatedly receive and send shallow text messages. It could be like the virtual reality shown in the movie The Matrix.

There is another interpretation of the merger of logos and pathos. Animals lack any distinction of logos and pathos; rather, the two are tightly merged. Therefore, the merger of logos and pathos might mean that human beings will revert to an animallike state. This would another bad outcome, in which we return to a very ancient time when human beings was not yet well developed and our behaviors were almost the same as those of other animals.

Instead, we probably should learn from our history. In both the Western world and the Asian world, there were eras when logos and pathos were not so clearly separated, 
yet people used to live fully human lives. For example, in the era of Homer's Iliad [5], logos and pathos were not clearly separated but the characters behaved honestly, bravely, and heroically. We should compare the behaviors of heroes and heroines in classical antiquity with those of modern people and determine from such comparisons what our behaviors should be in the future.

On the other hand, in Japan we have The Tale of Genji [6], the world's oldest novel. In once sense, the behaviors of the heroes and heroines in this novel are somewhat similar to those of current, younger generations. In the classic novel, men and women frequently exchange short poems, called waka, as a method of communication. Similarly, young people today frequently exchange short messages or post to Twitter accounts by using mobile phones. Probably the biggest difference, then, between these ancient and modern behaviors, is that the exchange of poems is a form of art creation, whereas message exchange using e-mail and Twitter does not involve art. The major question is how we can ennoble the communication behaviors of people in the network age. A good outcome would be to achieve the way of life described in The Tale of Genji but over a network using new media.

\section{Conclusion}

Various types of new entertainment, such as games and mobile phone applications, have been introduced into our society, rapidly changing our lifestyle. It seems we are in the era of chaos, and it is not certain what kind of lifestyle and society we will have in the future. In this situation, it is important to carefully observe the phenomena happening around us and extract findings, trends, tendencies, and so forth.

In this paper, by trying to answer the question of what entertainment is, I have tried to clarify the basic trends underlying various surface phenomena. It was first clarified that human behavior consists of two aspects, the logical and the emotional, and that entertainment is closely related to the emotional aspect.

Then, I discussed how Plato tried to separate the logical and emotional aspects of human behavior. Plato's efforts originated the long history of Western philosophy. Because entertainment is closely connected to the emotional side of behavior, it has been considered an informal aspect of behavior and thus hidden, even though entertainment is an essential aspect of our lives, like eating and sleeping.

It was also pointed out that the recent development of information and media technologies has raised the importance of the emotional aspect of life. In this sense, we are experiencing an entertainment renaissance. On the other hand, this means that logos and pathos, which have been clearly separated through history, are approaching each other and could even merge together.

There are several dangers in this trend. One is that the cultural differences that have enriched human history might fade away. Another is that human behaviors could revert to those of animals.

So far, there are no clear solutions to overcoming these dangers. One way to address this difficult situation, however, is to look back and learn from our history. There have been several eras when logos and pathos were joined together, as in the times described in the Iliad and The Tale of Genji. By learning from the behaviors detailed in these famous classics, we could perhaps imagine the future to which we are headed. 


\section{References}

1. http://www.weforum.org/en/index.htm

2. Ptato: The Rebublic. Penguin Classics

3. Tosa, N.: Cultural Computing. NTT Publishing Co. (2009)

4. Nakatsu, R.: Future of Communications Empowered by Technologies. Ohm-sha publishing Co. (2010)

5. Homer: Ilias. Hackett Publishing Company, Inc., Indianapolis (1997)

6. Murasaki, S.: The Tale of Genji. A Divition of Random House, Inc. (1976) 